\title{
WEIGHT-ALMOST GREEDY BASES
}

\author{
S. J. DILWORTH, DENKA KUTZAROVA, VLADIMIR TEMLYAKOV, \\ AND BEN WALLIS
}

\begin{abstract}
We introduce the notion of a weight-almost greedy basis and show that a basis for a real Banach space is $w$-almost greedy if and only if it is both quasi-greedy and $w$-democratic. We also introduce the notion of weight-semi-greedy basis and show that a $w$-almost greedy basis is $w$-semi-greedy and that the converse holds if the Banach space has finite cotype.
\end{abstract}

\section{INTRODUCTION}

Let $\left(e_{n}\right)_{n=1}^{\infty}$ be a normalized basis for a Banach space $X$ with biorthogonal functionals $\left(e_{n}^{*}\right)_{n=1}^{\infty}$. Konyagin and Temlyakov defined [KT] the thresholding greedy algorithm (TGA) for $\left(e_{n}\right)_{n=1}^{\infty}$ as the sequence $\left(G_{m}\right)_{m=1}^{\infty}$ of functions $G_{m}: X \rightarrow X$ by letting $G_{m}(x)$ denote the vector obtained by taking the $m$ largest coefficients of $x=\sum e_{n}^{*}(x) e_{n} \in X$. The TGA provides a theoretical model for the thresholding procedure that is used in image compression.

They defined the basis $\left(e_{n}\right)$ to be greedy if the TGA is optimal in the sense that $G_{n}(x)$ is essentially the best $n$-term approximation to $x$ using the basis vectors, i.e. if there exists a constant $K$ such that for all $x \in X$ and $m \in \mathbb{N}$, we have

$$
\left\|x-G_{m}(x)\right\| \leqslant K \inf \left\{\left\|x-\sum_{n \in A} \alpha_{n} e_{n}\right\|:|A|=m, \alpha_{n} \in \mathbb{R}, n \in A\right\} .
$$

They then showed that greedy bases can be simply characterized as unconditional bases with the additional property of being democratic, i.e. for some $\Delta>0$, we have

$$
\left\|\sum_{n \in A} e_{n}\right\| \leqslant \Delta\left\|\sum_{n \in B} e_{n}\right\| \quad \text { whenever }|A| \leqslant|B| .
$$

The first author was supported by the National Science Foundation under Grant Number DMS-1361461; the third author was supported by the Russian Federation Government Grant No. 14.W03.31.0031. The first and second authors were supported by the Workshop in Analysis and Probability at Texas A\&M University in 2017. 
They also defined a basis to be quasi-greedy if there exists a constant $K$ such that $\left\|G_{m}(x)\right\| \leqslant K\|x\|$ for all $x \in X$ and $m \in \mathbb{N}$. Subsequently, Wojtaszczyk [W] proved that these are precisely the bases for which the TGA merely converges, i.e. $\lim _{m \rightarrow \infty} G_{m}(x)=x$ for $x \in X$.

The class of almost greedy bases was introduced in [DKKT]. Let us denote the biorthogonal sequence by $\left(e_{n}^{*}\right)$. Then $\left(e_{n}\right)$ is almost greedy if there is a constant $K$ such that

$\left\|x-G_{m}(x)\right\| \leqslant K \inf \left\{\left\|x-\sum_{n \in A} e_{n}^{*}(x) e_{n}\right\|:|A|=m\right\} \quad x \in X, m \in \mathbb{N}$.

It was proved in [DKKT] that $\left(e_{n}\right)$ is almost greedy if and only if $\left(e_{n}\right)$ is quasi-greedy and democratic.

The class of semi-greedy bases was introduced in [DKK]. A basis is semi-greedy if there is a constant $C$ such that for all $x \in X$ and $m \in \mathbb{N}$

$$
\left\|x-\bar{G}_{m}(x)\right\| \leqslant C \inf \left\{\left\|x-\sum_{n \in A} \alpha_{n} e_{n}\right\|:|A|=m, \alpha_{n} \in \mathbb{R}, n \in A\right\},
$$

where $\bar{G}_{m}(x)$ is the best $m$ - term approximation to $x$ with the same (or possibly smaller) support as $G_{m}(x)$ (i.e., $\bar{G}_{m}(x)$ is supported on the basis vectors corresponding to the $m$ largest coefficients of $x$ ). It was proved in DKK that an almost greedy basis is semi-greedy and that the converse holds if $X$ has finite cotype.

Later, Kerkyacharian, Picard and Temlyakov [KPT] (see also [T1], Section 1.3) defined the notion of greedy basis with respect to a weight function, weight-greedy basis, and proved a criterion similar to the one for greedy bases. Their generalization was inspired by research of Cohen, DeVore and Hochmuth [CDH]. Recently, Berna and Blasco [BB] showed that greedy bases can be characterized as those where the error term using $m$-greedy approximant is uniformly bounded by the best $m$-term approximation with respect to polynomials with constant coefficients in the context of the weak greedy algorithm with weights.

In this paper we introduce the notion of a weight-almost greedy basis and show that a basis for a real Banach space is $w$-almost greedy if and only if it is both quasi-greedy and $w$-democratic.

From here onward, a weight $w=\left(w_{i}\right)_{i=1}^{\infty}$ will always be a sequence of positive real numbers. If $A \subset \mathbb{N}$ then we let $w(A)=\sum_{i \in A} w_{i}$ denote the $\boldsymbol{w}$-measure of $A$.

Let us give a more precise definition of each $G_{m}(x)$. If $\left(a_{i}\right)_{i=1}^{\infty} \in c_{0}$ then we denote by $\left(a_{i}^{*}\right)_{i=1}^{\infty}$ the nonincreasing rearrangement of $\left(\left|a_{i}\right|\right)_{i=1}^{\infty}$. Let $\tau$ be any permutation of $\mathbb{N}$ satisfying $\left|a_{\tau(i)}\right|=a_{i}^{*}$ for all $i \in \mathbb{N}$. 
Then $G_{m, \tau}(x)=\sum_{i=1}^{m} x_{\tau(i)}^{*}(x) x_{\tau(i)}$. We next set

$$
\Lambda_{m, \tau, x}:=\{\tau(1), \cdots, \tau(m)\},
$$

the set of indices of the coefficients associated with $G_{m, \tau}(x)$, called the support of $G_{m, \tau}(x)$. When $\tau$ is understood (or irrelevant) we write $G_{m}=G_{m, \tau}$ and $\Lambda_{m}=\Lambda_{m, \tau, x}$, and when $m$ and $x$ are also understood we write $\Lambda=\Lambda_{m, \tau, x}$.

As is usual, we write

$$
\mathbb{N}^{m}=\{A \subset \mathbb{N}:|A|=m\} \quad \text { and } \quad \mathbb{N}^{<\infty}=\bigcup_{m=0}^{\infty} \mathbb{N}^{m}
$$

Throughout, $C$ and $D$ will denote constants in $[1, \infty)$.

Definition 1.1. We say that a normalized basis $\left(e_{n}\right)_{n=1}^{\infty}$ for a Banach space $X$ is $\boldsymbol{w}$-almost greedy with constant $\boldsymbol{K}$ whenever

$$
\left\|x-G_{m}(x)\right\| \leqslant K \inf \left\{\left\|x-\sum_{n \in A} e_{n}^{*}(x) e_{n}\right\|: A \in \mathbb{N}^{<\infty}, w(A) \leqslant w(\Lambda)\right\}
$$

for all $m \in \mathbb{N}$ and $x \in X$, and independent of choice of $\tau$.

Remark 1.2. In the above definition we can always take $A=\emptyset$ to obtain

$$
\inf \left\{\left\|x-\sum_{n \in A} e_{n}^{*}(x) e_{n}\right\|: A \in \mathbb{N}^{<\infty}, w(A) \leqslant w(\Lambda)\right\} \leqslant\|x\| .
$$

Definition 1.3. A normalized basis $\left(e_{n}\right)_{n=1}^{\infty}$ is $\boldsymbol{w}$-democratic with constant $\boldsymbol{D}$ whenever $w(A) \leqslant w(B)$ for $A, B \in \mathbb{N}<\infty$ implies

$$
\left\|\sum_{n \in A} e_{n}\right\| \leqslant D\left\|\sum_{n \in B} e_{n}\right\|
$$

Remark 1.4. Observe that a subsequence $\left(x_{n_{k}}\right)_{k=1}^{\infty}$ of a $D$-w-democratic sequence $\left(e_{n}\right)_{n=1}^{\infty}$ is $D$ - $w^{\prime}$-democratic, where $w^{\prime}=\left(w_{n_{k}}\right)_{k=1}^{\infty}$ is the corresponding subsequence of $w$. Indeed, if $A \in \mathbb{N}^{<\infty}$ then we write $A^{\prime}=\left\{n_{a}: a \in A\right\}$ so that $w^{\prime}(A)=w\left(A^{\prime}\right)$.

When $w=(1,1,1, \cdots)$, we have $w(A)=|A|$ for each $A \in \mathbb{N}<\infty$. In this case, a $w$-almost greedy (resp., $w$-democratic) basis is called, simply, almost greedy (resp., democratic).

Finally, let us remark that we will often be forced to work with real Banach spaces, as most of the proofs are invalid in the complex setting. 
Lemma 2.1. Every $K$-w-almost greedy basis is $(K+1)$-quasi-greedy.

Proof. Fix $m$ and $x$. By Remark 1.2 together with $K$-w-almost-greediness,

$$
\left\|x-G_{m}(x)\right\| \leqslant K\|x\|
$$

and hence

$$
\left\|G_{m}(x)\right\| \leqslant\left\|x-G_{m}(x)\right\|+\|x\| \leqslant(K+1)\|x\| .
$$

Let us give two facts from previous literature, which will be required momentarily.

Proposition 2.2 ([DKKT, Lemma 2.1]). If $\left(e_{n}\right)_{n=1}^{\infty}$ is a K-quasigreedy basis for a real Banach space then

$$
\frac{1}{2 K}\left\|\sum_{n \in A} e_{n}\right\| \leqslant\left\|\sum_{n \in A} \varepsilon_{n} e_{n}\right\| \leqslant 2 K\left\|\sum_{n \in A} e_{n}\right\|
$$

for every choice of signs $\left(\varepsilon_{n}\right)_{n=1}^{\infty} \in\{ \pm 1\}^{\mathbb{N}}$ and every $A \in \mathbb{N}<\infty$. In this case we have

$$
\left\|\sum_{n \in A} a_{n} e_{n}\right\| \leqslant 2 K\left\|\sum_{n \in A} e_{n}\right\| \cdot\left\|\left(a_{n}\right)_{n \in A}\right\|_{\infty}
$$

for every $\left(a_{n}\right)_{n=1}^{\infty} \in \mathbb{R}^{\mathbb{N}}$ and $A \in \mathbb{N}^{<\infty}$.

Proposition 2.3 ([DKKT, Lemma 2.2]). If $\left(e_{n}\right)_{n=1}^{\infty}$ is a K-quasigreedy basis for a real Banach space then

$$
\left\|\sum_{n \in A} e_{n}\right\| \cdot \min _{n \in A}\left|a_{n}\right| \leqslant 4 K^{2}\left\|\sum_{n \in A} a_{n} e_{n}\right\|
$$

for every $\left(a_{n}\right)_{n=1}^{\infty} \in \mathbb{R}^{\mathbb{N}}$ and $A \in \mathbb{N}^{<\infty}$.

The reader can also find the above Propositions in [T2], p.65.

Theorem 2.4. Every normalized $K$-w-almost greedy basis for a real Banach space is $w$-democratic with constant $\leqslant K$.

Proof. Let $A, B \subset \mathbb{N}<\infty$ satisfy $w(A) \leqslant w(B)$. Note that this also means $w(A \backslash B) \leqslant w(B \backslash A)$. For $\delta>0$ we set

$$
\left.y:=\sum_{n \in A} e_{n}+(1+\delta) \sum_{n \in B \backslash A} e_{n} \quad \text { and } \quad m:=|B \backslash A|\right) .
$$


By $K$-w-almost-greediness, we now have the estimates

$$
\begin{aligned}
\left\|\sum_{n \in A} e_{n}\right\| & =\left\|y-(1+\delta) \sum_{n \in B \backslash A} e_{n}\right\| \\
& =\left\|y-G_{m}(y)\right\| \\
& \leqslant K\left\|y-\sum_{n \in A \backslash B} e_{n}\right\| \\
& =K\left\|\sum_{n \in B} e_{n}+\delta \sum_{n \in B \backslash A} e_{n}\right\| \\
& \leqslant K\left\|\sum_{n \in B} e_{n}\right\|+\delta\left\|\sum_{n \in B \backslash A} e_{n}\right\| .
\end{aligned}
$$

As $\delta>0$ was arbitrary, we have

$$
\left\|\sum_{n \in A} e_{n}\right\| \leqslant K\left\|\sum_{n \in B} e_{n}\right\|
$$

Theorem 2.5. If a basis $\left(e_{n}\right)_{n=1}^{\infty}$ for a real Banach space is both $K$ quasi-greedy and D-w-democratic, then it is $w$-almost greedy with constant $\leqslant 8 K^{4} D+K+1$.

Proof. Fix $m$ and $x$, and suppose $A \in \mathbb{N}<\infty$ satisfies $w(A) \leqslant w(\Lambda)$. We claim that

$$
\left\|x-G_{m}(x)\right\| \leqslant\left[4 K^{3}(K+1) D+K+1\right] \cdot\left\|x-\sum_{n \in A} e_{n}^{*}(x) e_{n}\right\|,
$$

so that, taking the infimum over all such $A$, the proof will be complete.

We begin with the estimate

$$
\begin{aligned}
\left\|x-G_{m}(x)\right\| & =\left\|x-\sum_{n \in \Lambda} e_{n}^{*}(x) e_{n}\right\| \\
& \leqslant\left\|x-\sum_{n \in A} e_{n}^{*}(x) e_{n}\right\|+\left\|\sum_{n \in A} e_{n}^{*}(x) e_{n}-\sum_{n \in \Lambda} e_{n}^{*}(x) e_{n}\right\| \\
& =\left\|x-\sum_{n \in A} e_{n}^{*}(x) e_{n}\right\|+\left\|\sum_{n \in A \backslash \Lambda} e_{n}^{*}(x) e_{n}-\sum_{n \in \Lambda \backslash A} e_{n}^{*}(x) e_{n}\right\| \\
& \leqslant\left\|x-\sum_{n \in A} e_{n}^{*}(x) e_{n}\right\|+\left\|\sum_{n \in A \backslash \Lambda} e_{n}^{*}(x) e_{n}\right\|+\left\|\sum_{n \in \Lambda \backslash A} e_{n}^{*}(x) e_{n}\right\| .
\end{aligned}
$$


Observe that $w(A \backslash \Lambda) \leqslant w(\Lambda \backslash A)$. Thus,

$$
\begin{aligned}
\left\|\sum_{n \in A \backslash \Lambda} e_{n}^{*}(x) e_{n}\right\| & \leqslant 2 K\left\|\sum_{n \in A \backslash \Lambda} e_{n}\right\| \cdot\left\|\left(e_{n}^{*}(x)\right)_{n \in A \backslash \Lambda}\right\|_{\infty} & & \text { (Proposition 2.2) } \\
& \leqslant 2 K D\left\|\sum_{n \in \Lambda \backslash A} e_{n}\right\| \cdot\left\|\left(e_{n}^{*}(x)\right)_{n \in A \backslash \Lambda}\right\|_{\infty} & & (D-w \text {-democracy) } \\
& \leqslant 2 K D\left\|\sum_{n \in \Lambda \backslash A} e_{n}\right\| \cdot \min _{n \in \Lambda \backslash A}\left|e_{n}^{*}(x)\right| & & \text { (definition of } \Lambda) \\
& \leqslant 8 K^{3} D\left\|\sum_{n \in \Lambda \backslash A} e_{n}^{*}(x) e_{n}\right\| & & \text { (Proposition 2.3). }
\end{aligned}
$$

Combining this with the last inequality gives

$$
\left\|x-G_{m}(x)\right\| \leqslant\left\|x-\sum_{n \in A} e_{n}^{*}(x) e_{n}\right\|+\left(8 K^{3} D+1\right)\left\|\sum_{n \in \Lambda \backslash A} e_{n}^{*}(x) e_{n}\right\| .
$$

Finally, we have

$$
\sum_{n \in \Lambda \backslash A} e_{n}^{*}(x) e_{n}=G_{s}\left(x-\sum_{n \in A} e_{n}^{*}(x) e_{n}\right), \quad s:=|\Lambda \backslash A|,
$$

and therefore, by $K$-quasi-greediness,

$$
\left\|\sum_{n \in \Lambda \backslash A} e_{n}^{*}(x) e_{n}\right\| \leqslant K\left\|x-\sum_{n \in A} e_{n}^{*}(x) e_{n}\right\| .
$$

We combine this with (2.1) to obtain

$$
\left\|x-G_{m}(x)\right\| \leqslant\left(8 K^{4} D+K+1\right)\left\|x-\sum_{n \in A} e_{n}^{*}(x) e_{n}\right\| .
$$

Theorem 2.6. A basis for a real Banach space is w-almost greedy if and only if it is both quasi-greedy and w-democratic.

Remark 2.7. Our proofs use only the following property of the $w$ measure:

$$
w(\emptyset)=0 \quad \text { and } \quad w(A) \leqslant w(B) \Rightarrow w(A \backslash B) \leqslant w(B \backslash A) .
$$

This property corresponds to a more general case than the one given by a $w$-measure. Indeed, here is an example of a strictly monotone set function $\nu$ defined on all finite subsets of $\mathbb{N}$ satisfying Property (*):

$$
\nu(\emptyset)=0 \quad \text { and } \quad \nu(A) \leqslant \nu(B) \Rightarrow \nu(A \backslash B) \leqslant \nu(B \backslash A)
$$


for which there does not exist a weight $w$ such that

$$
\nu(A) \leqslant \nu(B) \Leftrightarrow w(A) \leqslant w(B) .
$$

First we define $\nu$ on the power set of $\{1,2,3\}$ by $\nu(\{1\})=\nu(\{2\})=$ $\nu(\{3\})=1 / 4, \nu(\{1,2\})=5 / 16, \nu(\{2,3\})=3 / 8, \nu(\{1,3\})=7 / 16$, $\nu(\{1,2,3\})=1 / 2$. It is easily seen that $\nu$ is strictly monotone and has Property $(*)$ (restricted to subsets of $\{1,2,3\}$ ).

Note that $\nu$ is not equivalent to a weight $w$ because $\nu(\{1\})=\nu(\{2\})=$ $\nu(\{3\})$ would imply by (2.2) that $w_{1}=w_{2}=w_{3}$ which would imply that $w(\{1,2\})=w(\{1,3\})=w(\{2,3\})$ which in turn would imply by (2.2) that $\nu(\{1,2\})=\nu(\{1,3\})=\nu(\{2,3\})$.

Now we extend $\nu$ to all finite subsets $A$ of $\mathbb{N}$. Write $A=A_{1} \cup A_{2}$ where $A_{1}=A \cap\{1,2,3\}, A_{2}=A \backslash A_{1}$. Define $\nu(A)=\nu\left(A_{1}\right)+\left|A_{2}\right|$. Clearly $\nu$ is strictly monotone.

Let us check Property $\left(^{*}\right)$. Suppose $\nu(A) \leqslant \nu(B)$, where $A=A_{1} \cup A_{2}$ and $B=B_{1} \cup B_{2}$. Then $\nu\left(A_{1}\right)+\left|A_{2}\right| \leqslant \nu\left(B_{1}\right)+\left|B_{2}\right|$. Now $\mid \nu\left(A_{1}\right)-$ $\nu\left(B_{1} \mid \leqslant 1 / 2\right.$ and hence $\left|A_{2}\right| \leqslant\left|B_{2}\right|$. Also $\nu(A \backslash B)=\nu\left(A_{1} \backslash B_{1}\right)+\mid A_{2} \backslash$ $B_{2} \mid$ and $\nu(B \backslash A)=\nu\left(B_{1} \backslash A_{1}\right)+\left|B_{2} \backslash A_{2}\right|$.

We consider two cases. First, if $\left|A_{2}\right|=\left|B_{2}\right|$, then $\nu\left(A_{1}\right) \leqslant \nu\left(B_{1}\right)$ and hence $\nu\left(A_{1} \backslash B_{1}\right) \leqslant \nu\left(B_{1} \backslash A_{1}\right)$ since $A_{1}, B_{1} \subseteq\{1,2,3\}$. But also $\left|A_{2} \backslash B_{2}\right|=\left|B_{2} \backslash A_{2}\right|$ and hence $\nu(A \backslash B) \leqslant \nu(B \backslash A)$.

Secondly, if $\left|A_{2}\right| \leqslant\left|B_{2}\right|-1$, then $\left|A_{2} \backslash B_{2}\right| \leqslant\left|B_{2} \backslash A_{2}\right|-1$ and hence $\nu\left(A_{1} \backslash B_{1}\right)+\left|A_{2} \backslash B_{2}\right| \leqslant 1 / 2+\left|B_{2} \backslash A_{2}\right|-1<\nu\left(B_{1} \backslash A_{1}\right)+\left|B_{2} \backslash A_{2}\right|$, and hence $\nu(A \backslash B) \leqslant \nu(B \backslash A)$.

\section{LEBESGUE-TYPE INEQUALITIES}

For $x \in X$ and $m \in \mathbb{N}$ denote

$$
\tilde{\sigma}_{m}(x):=\inf \left\{\left\|x-\sum_{n \in A} e_{n}^{*}(x) e_{n}\right\|:|A|=m\right\}
$$

the expansional best $m$-term approximation of $x$ with respect to $E:=$ $\left\{e_{n}\right\}_{n=1}^{\infty}$. The following Lebesgue-type inequality was obtained in [DKKT]: suppose $E$ is $K$-quasi-greedy and $D$-democratic, then

$$
\left\|x-G_{m}(x)\right\| \leqslant C(K) D \tilde{\sigma}_{m}(x) .
$$

This inequality was slightly generalized in [T2], p.91, Theorem 3.5.2. Suppose that for a basis $E$ there exists an increasing sequence $v(m):=$ $v(m, E)$ such that, for any two sets of indices $A$ and $B$ with $|A| \leqslant$ $|B| \leqslant m$, we have

$$
\left\|\sum_{n \in A} e_{n}\right\| \leqslant v(m)\left\|\sum_{n \in B} e_{n}\right\| .
$$


The following statement is from [T2], p.91, Theorem 3.5.2.

Proposition 3.1. Let $E$ be a $K$-quasi-greedy basis of $X$ satisfying (3.1). Then, for each $x \in X$,

$$
\left\|x-G_{m}(x)\right\| \leqslant C(K) v(m) \tilde{\sigma}_{m}(x) .
$$

The above proof of Theorem 2.5 gives us the following version of Proposition 3.1 in the weighted case. Suppose that for given weight sequence $w=\left\{w_{n}\right\}_{n=1}^{\infty}$ and basis $E$ there exists an increasing function $v(u, w):=v(u, E, w)$ such that, for any two sets of indices $A$ and $B$ with $w(A) \leqslant w(B) \leqslant u$, we have

$$
\left\|\sum_{n \in A} e_{n}\right\| \leqslant v(u, w)\left\|\sum_{n \in B} e_{n}\right\|
$$

For $x \in X$ and $u>0$ denote for a given weight sequence $w$

$$
\tilde{\sigma}_{u}^{w}(x):=\inf \left\{\left\|x-\sum_{n \in A} e_{n}^{*}(x) e_{n}\right\|: w(A) \leqslant u\right\} .
$$

Theorem 3.2. Let $E$, a $K$-quasi-greedy basis of $X$, and a weight sequence $w$ satisfy (3.2). Then, for each $x \in X$,

$$
\left\|x-G_{m}(x)\right\| \leqslant C(K) v\left(w\left(\Lambda_{m}\right), w\right) \tilde{\sigma}_{w\left(\Lambda_{m}\right)}^{w}(x),
$$

where $\Lambda_{m}$ is from

$$
G_{m}(x)=\sum_{n \in \Lambda_{m}} e_{n}^{*}(x) e_{n} .
$$

We note that the left hand side of (3.3) does not depend on the weight sequence $w$, but the right hand side of (3.3) does depend on $w$. Therefore, our setting with weights allows us to estimate $\left\|x-G_{m}(x)\right\|$ choosing different weight sequences $w$ and optimize over them.

Theorem 3.2 gives a Lebesgue-type inequality in terms of expansional best $m$-term approximation. We now consider a question of replacing the expansional best $m$-term approximation by the best $m$-term approximation

$$
\sigma_{u}^{w}(x):=\inf \left\{\left\|x-\sum_{n \in A} c_{n} e_{n}\right\|: c_{n}, n \in A, w(A) \leqslant u\right\}
$$

where inf is taken over all sets of indices $A$ with $w$-measure $w(A) \leqslant u$ and all coefficients $c_{n}, n \in A$. In case $w_{i}=1, i \in \mathbb{N}$, we drop $w$ from the notation. It is clear that

$$
\sigma_{u}^{w}(x, E) \leqslant \tilde{\sigma}_{u}^{w}(x, E) .
$$

It is also clear that for an unconditional basis $E$ we have

$$
\tilde{\sigma}_{u}^{w}(x, E) \leqslant C(X, E) \sigma_{m}^{w}(x, E) .
$$


We recall some known useful properties of quasi-greedy bases. For a given element $x \in X$ we consider the expansion

$$
x=\sum_{k=1}^{\infty} e_{k}^{*}(x) e_{k} .
$$

Let a sequence $k_{j}, j=1,2, \ldots$, of positive integers be such that

$$
\left|e_{k_{1}}^{*}(x)\right| \geqslant\left|e_{k_{2}}^{*}(x)\right| \geqslant \ldots
$$

We use the notation

$$
a_{j}(x):=\left|e_{k_{j}}^{*}(x)\right|
$$

for the decreasing rearrangement of the coefficients of $x$. It will be convenient in this section to redefine the quasi-greedy constant $K$ to be the least constant such that

$$
\left\|G_{m}(f)\right\| \leqslant K\|f\| \quad \text { and } \quad\left\|f-G_{m}(f)\right\| \leqslant K\|f\|, \quad f \in X .
$$

The following Lemma 3.3 is from [DKKT] (see also [T2], p.66).

Lemma 3.3. Suppose $E=\left\{e_{n}\right\}_{n \in \mathbb{N}}$ has quasi-greedy constant $K$. For any $x \in X$ and any $n \in \mathbb{N}$ we have

$$
a_{n}(x)\left\|\sum_{j=1}^{n} e_{k_{j}}\right\| \leqslant 4 K^{2}\|x\| .
$$

For a set of indices $\Lambda$ define

$$
S_{\Lambda}(x, E):=\sum_{k \in \Lambda} e_{k}^{*}(x) e_{k} .
$$

The following Lemma 3.4 is from [DKK] (see also [T2], p.67).

Lemma 3.4. Let $E$ be a quasi-greedy basis of $X$. Then for any finite set of indices $\Lambda,|\Lambda|=m$, we have for all $x \in X$

$$
\left\|S_{\Lambda}(x, E)\right\| \leqslant C \ln (m+1)\|x\| .
$$

Lemma 3.4 was used in [GHO] and [DS-BT] to prove the following inequality.

Lemma 3.5. Let $E$ be a quasi-greedy basis of $X$. Then for all $x \in X$

$$
\tilde{\sigma}_{m}(x) \leqslant C \ln (m+1) \sigma_{m}(x) .
$$

We prove an estimate for $\tilde{\sigma}_{n}^{w}(x, E)$ in terms of $\sigma_{m}^{w}(x, E)$ for a quasigreedy basis $E$. For a basis $E$ we define the fundamental function

$$
\varphi^{w}(u):=\sup _{A: w(A) \leqslant u}\left\|\sum_{k \in A} e_{k}\right\| .
$$


We also need the following function

$$
\phi^{w}(u):=\inf _{A: w(A)>u}\left\|\sum_{k \in A} e_{k}\right\| .
$$

In the case $w_{i}=1, i \in \mathbb{N}$, we drop $w$ from the notation. The following inequality was obtained in [DKKT].

Lemma 3.6. Let $E$ be a quasi-greedy basis. Then for any $m$ and $r$ there exists a set $F,|F| \leqslant m+r$ such that

$$
\left\|x-S_{F}(x)\right\| \leqslant C\left(1+\frac{\varphi(m)}{\phi(r)}\right) \sigma_{m}(x)
$$

and, therefore, for any $x \in X$

$$
\tilde{\sigma}_{m+r}(x) \leqslant C\left(1+\frac{\varphi(m)}{\phi(r)}\right) \sigma_{m}(x) .
$$

We now prove the following weighted version of Lemma 3.6.

Lemma 3.7. Let $E$ be a quasi-greedy basis. Then for any $u$ and $v$ we have for each $x \in X$

$$
\tilde{\sigma}_{u+v}^{w}(x) \leqslant C\left(1+\frac{\varphi(u)}{\phi(v)}\right) \sigma_{u}^{w}(x) .
$$

Proof. For an arbitrary $\varepsilon>0$, let $A$ be a set, $w(A) \leqslant u$, and $p_{u}(x)$ be a polynomial such that

$$
\left\|x-p_{u}(x)\right\| \leqslant \sigma_{u}^{w}(x)+\varepsilon, \quad p_{u}(x)=\sum_{k \in A} b_{k} e_{k} .
$$

Denote $g:=x-p_{u}(x)$. Let $m$ be such that for the set $B:=\Lambda_{m}(g)$ we have $w(B) \leqslant v$ and $w\left(\Lambda_{m+1}(g)\right)>v$. Consider

$$
G_{m}(g)=\sum_{k \in B} e_{k}^{*}(g) e_{k}
$$

We have

$$
x-S_{A \cup B}(x)=g-S_{A \cup B}(g)=g-S_{B}(g)-S_{A \backslash B}(g) .
$$

By the assumption that $E$ is quasi-greedy and by the definition of $B$ we get

$$
\left\|g-S_{B}(g)\right\| \leqslant C_{1}\|g\| \leqslant C_{1}\left(\sigma_{u}^{w}(x)+\varepsilon\right) .
$$

Let us estimate $\left\|S_{A \backslash B}(g)\right\|$. By Lemma 3.3 we get

$$
\max _{k \in A \backslash B}\left|e_{k}^{*}(g)\right| \leqslant a_{m+1}(g) \leqslant 4 K^{2}(\phi(v))^{-1}\|g\| .
$$

Next, by Proposition 2.2 we obtain

$$
\left\|S_{A \backslash B}(g)\right\| \leqslant(2 K)^{3} \varphi(u) \phi(v)^{-1}\|g\| .
$$


Combining (3.7) and (3.8) we derive from (3.6) for $F:=A \cup B$

$$
\left\|f-S_{F}(x)\right\| \leqslant C(K)\left(1+\frac{\varphi(u)}{\phi(v)}\right)\left(\sigma_{u}^{w}(x)+\varepsilon\right) .
$$

Lemma 3.7 is proved.

Theorem 3.8. Let $E$ be a K-w-quasi-greedy and $D$-w-democratic. Then, for any $x \in X$, we have

$$
\left\|x-G_{m}(x)\right\| \leqslant C(K, D) \sigma_{w\left(\Lambda_{m}\right) / 2}^{w}(x) .
$$

Proof. By Theorem 2.5 we have

$$
\left\|x-G_{m}(x)\right\| \leqslant C(K) D \tilde{\sigma}_{w\left(\Lambda_{m}\right)}^{w}(x) .
$$

Using inequality $\varphi^{w}(u) \leqslant D \phi^{w}(u)$, by Lemma 3.7 with $u=v=$ $w\left(\Lambda_{m}\right) / 2$ we obtain

$$
\tilde{\sigma}_{w\left(\Lambda_{m}\right)}^{w}(x) \leqslant C(K)(1+D) \sigma_{w\left(\Lambda_{m}\right) / 2}^{w}(x) .
$$

Combining (3.9) and (3.10) we complete the proof of Theorem 3.8.

\section{4. $w$-SEMI-GREEDY BASES}

In this section we consider an obvious enhancement of the TGA which improves the rate of convergence. Suppose that $x \in X$ and let $\rho$ be the greedy ordering for $x$. Let $\bar{G}_{m}(x) \in \operatorname{span}\left\{e_{\rho(n)}: 1 \leqslant n \leqslant m\right\}$ be a Chebyshev approximation to $x$. Thus,

$$
\left\|x-\bar{G}_{m}(x)\right\|=\min \left\{\left\|x-\sum_{n=1}^{m} a_{n} e_{\rho(n)}\right\|:\left(a_{n}\right)_{n=1}^{m} \in \mathbb{R}^{m}\right\} .
$$

For $y>0$, let

$$
\sigma_{y}^{w}(x):=\inf \left\{\left\|x-\sum_{n \in A} a_{n} e_{n}\right\|: w(A) \leqslant y, a_{n} \in \mathbb{R}\right\}
$$

denote the error in the best approximation to $x$ by vectors of support of weight at most $y$.

Definition 4.1. We say that a basis $\left(e_{n}\right)_{n=1}^{\infty}$ for a Banach space $X$ is $w$-semi-greedy with constant $\bar{K}$ if

$$
\left\|x-\bar{G}_{m}(x)\right\| \leqslant \bar{K} \sigma_{w\left(\Lambda_{m}\right)}^{w}(x)
$$

for all $m \in \mathbb{N}$ and $x \in X$. 
Our first goal is to prove that every $w$-almost greedy basis is $w$-semigreedy, which generalizes [DKK, Theorem 3.2]. To that end, we recall an important property of the 'truncation function' proved in [DKK].

Fix $M>0$. Define the truncation function $f_{M}: \mathbb{R} \rightarrow[-M, M]$ thus:

$$
f_{M}(x)= \begin{cases}M & \text { for } x>M \\ x & \text { for } x \in[-M, M] \\ -M & \text { for } x<-M\end{cases}
$$

Proposition 4.2 ([DKK, Proposition 3.1]). Suppose that $\left(e_{n}\right)$ is $K$ quasi-greedy. Then, for every $M>0$ and for all real scalars $\left(a_{n}\right)$, we have

$$
\left\|\sum_{i=1}^{\infty} f_{M}\left(a_{n}\right) e_{n}\right\| \leqslant(1+3 K)\left\|\sum_{n=1}^{\infty} a_{n} e_{n}\right\| .
$$

Theorem 4.3. Every w-almost greedy basis of a real Banach space $X$ is w-semi-greedy.

Proof. By Lemma 2.1 and Theorem 2.4, $\left(e_{n}\right)$ is quasi-greedy and wdemocratic. Let $K$ and $D$ the quasi-greedy and democratic constants of $\left(e_{n}\right)$, respectively. Fix $m \geqslant 1$ and $x=\sum_{n=1}^{\infty} a_{n} e_{n}$ in $X$. Let $\Lambda:=$ $\Lambda_{m, \tau, x}$. Let $z:=\sum_{n \in B} b_{n} e_{n}$, where $w(B) \leqslant w(\Lambda)$, satisfy

$$
\|x-z\| \leqslant 2 \sigma_{w(\Lambda)}^{w}(x)
$$

If $B=\Lambda$ then there is nothing to prove. So we may assume (since $w(B) \leqslant w(\Lambda))$ that $\Lambda \backslash B$ is nonempty. Let $k=|\Lambda \backslash B|$, so that $1 \leqslant$ $k \leqslant m$, and let $M=\left|a_{\rho(m)}\right|$. Then, using both parts of Proposition 2.2.

$$
M\left\|\sum_{n \in \Lambda \backslash B} e_{n}\right\| \leqslant 2 K M\left\|\sum_{n \in \Lambda} e_{n}\right\| \leqslant 4 K^{2}\|x-z\|,
$$

since $\left|e_{n}^{*}(x-z)\right| \geqslant M$ for all $n \in \Lambda \backslash B$. Let

$$
x-z:=\sum_{n=1}^{\infty} y_{n} e_{n}
$$

By Proposition 4.2, we have

$$
\left\|\sum_{n=1}^{\infty} f_{M}\left(y_{n}\right) e_{n}\right\| \leqslant(1+3 K)\|x-z\| .
$$


Note that

$$
\begin{aligned}
v: & =\sum_{n \in \Lambda} f_{M}\left(y_{n}\right) e_{n}+\sum_{n \in \mathbb{N} \backslash \Lambda} a_{n} e_{n} \\
& =\sum_{n=1}^{\infty} f_{M}\left(y_{n}\right) e_{n}+\sum_{n \in B \backslash \Lambda}\left(a_{n}-f_{M}\left(y_{n}\right)\right) e_{n}
\end{aligned}
$$

since $a_{n}=y_{n}=f_{M}\left(y_{n}\right)$ for all $n \in \mathbb{N} \backslash(\Lambda \cup B)$ Hence,

$$
\begin{aligned}
\|v\| & \leqslant\left\|\sum_{n=1}^{\infty} f_{M}\left(y_{n}\right) e_{n}\right\|+\left\|\sum_{n \in B \backslash \Lambda}\left(a_{n}-f_{M}\left(y_{n}\right)\right) e_{n}\right\| \\
& \leqslant(1+3 K)\|x-z\|+4 K M\left\|\sum_{n \in B \backslash \Lambda} e_{n}\right\|
\end{aligned}
$$

(by (4.2) and by Proposition 2.2, since $\left|a_{n}-f_{M}\left(y_{n}\right)\right| \leqslant 2 M$ for all $n \in B \backslash \Lambda)$

$$
\leqslant(1+3 K)\|x-z\|+4 K D M\left\|\sum_{n \in \Lambda \backslash B} e_{n}\right\|
$$

(since $w(B \backslash \Lambda) \leqslant w(\Lambda \backslash B)$ and $\left(e_{n}\right)$ is $w$-democratic)

$$
\leqslant\left(1+3 K+16 K^{3} D\right)\|x-z\|
$$

by (4.1). Taking the infimum over all $z$ gives

$$
\|v\| \leqslant\left(1+3 K+16 K^{3} D\right) \sigma_{w(\Lambda)}^{w}(x)
$$

Since $v=x-\sum_{n \in \Lambda}\left(a_{n}-f_{M}\left(y_{n}\right)\right) e_{n}$, we conclude that $\left(e_{n}\right)$ is semigreedy with constant $1+3 K+16 K^{3} D$.

Corollary 4.4. Suppose $\left(e_{n}\right)$ is w-almost greedy. Then, for all $x \in X$ and $m \geqslant 1$,

$$
\left\|x-G_{m}(x)\right\| \leqslant\left(1+3 K+16 K^{3} D\right) \sigma_{w(\Lambda)}^{w}(x)+2 K\left|e_{\rho(m)}^{*}(x)\right|\left\|\sum_{n \in \Lambda} e_{n}\right\| .
$$

Proof. Using the notation of the last result, note that $x-G_{m}(x)=$ $v-\sum_{n \in \Lambda} f_{M}\left(y_{n}\right) e_{n}$. Hence

$$
\begin{aligned}
\left\|x-G_{m}(x)\right\| & \leqslant\|v\|+\left\|\sum_{n \in \Lambda} f_{M}\left(y_{n}\right) e_{n}\right\| \\
& \leqslant\left(1+3 K+16 K^{3} D\right)\|x-z\|+2 K\left|e_{\rho(m)}^{*}(x)\right|\left\|\sum_{n \in \Lambda} e_{n}\right\|,
\end{aligned}
$$


by Proposition 2.2 recalling that $M=\left|e_{\rho(m)}^{*}(x)\right|$. Now take the infimum of $\|x-z\|$ over $z$ to get the result.

The remainder of this section investigates the converse of Theorem 4.3. To that end we first show that certain properties of the weight sequence $\left(w_{n}\right)$ imply the existence of subsequences of $\left(e_{n}\right)$ that are equivalent to the unit vector basis of $c_{0}$.

Proposition 4.5. Let $\left(e_{n}\right)$ be a w-semi-greedy basis with constant $\bar{K}$ and let $\beta$ be the basis constant. Suppose $A \in \mathbb{N}<\infty$.

(i) If $w(A) \leqslant \lim \sup _{n \rightarrow \infty} w_{n}$ then $\max _{ \pm}\left\|\sum_{n \in A} \pm e_{n}\right\| \leqslant 2 \beta \bar{K}$.

(ii) If $\sum_{n=1}^{\infty} w_{n}<\infty$ then $\left(e_{n}\right)$ is equivalent to the unit vector basis of $c_{0}$.

(iii) If $\sup w_{n}=\infty$ then $\left(e_{n}\right)$ is equivalent to the unit vector basis of $c_{0}$.

(iv) If inf $w_{n}=0$ then $\left(e_{n}\right)$ contains a subsequence equivalent to the unit vector basis of $c_{0}$.

Proof. (i) We may select $n_{1}>n_{0}>\max A$ such that $w(A)<\delta:=$ $w_{n_{0}}+w_{n_{1}}$. Let $\varepsilon>0$. The $w$-semi-greedy condition applied to $x:=$ $\sum_{n \in A} \pm e_{n}+(1+\varepsilon)\left(e_{n_{0}}+e_{n_{1}}\right)$ implies the existence of $\lambda, \mu \in \mathbb{R}$ such that

$$
\left\|\sum_{n \in A} \pm e_{n}+\lambda e_{n_{0}}+\mu e_{n_{1}}\right\| \leqslant \bar{K} \sigma_{\delta}^{w}(x)
$$

Hence

$$
\begin{aligned}
\left\|\sum_{n \in A} \pm e_{n}\right\| & \leqslant \beta\left\|\sum_{n \in A} \pm e_{n}+\lambda e_{n_{0}}+\mu e_{n_{1}}\right\| \\
& \leqslant \beta \bar{K} \sigma_{\delta}^{w}(x) \\
& \leqslant \beta \bar{K}(1+\varepsilon)\left\|e_{n_{0}}+e_{n_{1}}\right\|
\end{aligned}
$$

(since $w(A) \leqslant \delta)$

$$
\leqslant 2 \beta \bar{K}(1+\varepsilon)
$$

Let $\varepsilon \rightarrow 0$ to conclude.

(ii) Choose $N \in \mathbb{N}$ such that $\sum_{N+1}^{\infty} w_{n}<w_{1}$. Suppose min $A \geqslant N+$ 1. The $w$-semi-greedy condition applied to $x:=(1+\varepsilon) e_{1}+\sum_{n \in A} \pm e_{n}$ implies the existence of $\lambda \in \mathbb{R}$ such that

$$
\left\|\lambda e_{1}+\sum_{n \in A} \pm e_{n}\right\| \leqslant \bar{K} \sigma_{w_{1}}^{w}(x)
$$


Hence

$$
\begin{aligned}
\left\|\sum_{n \in A} \pm e_{n}\right\| & \leqslant 2 \beta\left\|\lambda e_{1}+\sum_{n \in A} \pm e_{n}\right\| \\
& \leqslant 2 \beta \bar{K} \sigma_{w_{1}}^{w}(x) \\
& \leqslant 2 \beta \bar{K}(1+\varepsilon)\left\|e_{1}\right\|
\end{aligned}
$$

(since $\left.w(A) \leqslant w_{1}\right)$

$$
=2 \beta \bar{K}(1+\varepsilon) .
$$

Hence $\left(e_{n}\right)$ is equivalent to the unit vector basis of $c_{0}$.

(iii) By (i), $\left\|\sum_{n \in A} \pm e_{n}\right\| \leqslant 2 \beta \bar{K}$ for all $A$ and choices of signs. Hence $\left(e_{n}\right)$ is equivalent to the unit vector basis of $c_{0}$.

(iv) Choose $\left(n_{k}\right)$ such that $\sum_{k=1}^{\infty} w_{n_{k}}<\infty$. By (ii), $\left(e_{n_{k}}\right)$ is equivalent to the unit vector basis of $c_{0}$.

The following definition generalizes the notion of superdemocracy to weights, with the constant weight corresponding to the usual definition of superdemocracy (see $[\mathrm{KT}]$ ).

Definition 4.6. A basis $\left(e_{n}\right)_{n=1}^{\infty}$ is $\boldsymbol{w}$-superdemocratic with constant $\overline{\boldsymbol{D}}$ whenever $w(A) \leqslant w(B)$ for $A, B \in \mathbb{N}<\infty$ implies

$$
\max _{ \pm}\left\|\sum_{n \in A} \pm e_{n}\right\| \leqslant \bar{D} \min _{ \pm}\left\|\sum_{n \in B} \pm e_{n}\right\|
$$

Combining the fact that quasi-greedy sequences are 'unconditional for constant coefficients' (Proposition 2.2) with Theorem 2.6 immediately yields the following result.

Proposition 4.7. Every w-almost greedy basis of a real Banach space is w-superdemocratic.

We show next that $w$-semi-greedy bases are also $w$-superdemocratic, which is the weighted version of [DKK, Proposition 3.3].

Theorem 4.8. Every w-semi-greedy basis of a real Banach space is w-superdemocratic.

Proof. We may assume that $\sum w_{n}=\infty$ and $\sup w_{n}<\infty$, for otherwise by Proposition $4.5\left(e_{n}\right)$ is equivalent to the unit vector basis of $c_{0}$ for which the result is obvious. Suppose $w(A) \leqslant w(B)$ and that $B$ is nonempty. If $w(B) \leqslant \lim \sup w_{n}$ then by Proposition 4.5,

$$
\max _{ \pm}\left\|\sum_{n \in A} \pm e_{n}\right\| \leqslant 2 \beta \bar{K}
$$


and hence

$$
\max _{ \pm}\left\|\sum_{n \in A} \pm e_{n}\right\| \leqslant 2 \beta \bar{K} \min _{ \pm}\left\|\sum_{n \in B} \pm e_{n}\right\|
$$

as desired. Now suppose that $w(B)>\lim \sup w_{n}$. Since $\sum w_{n}=\infty$ we can choose $E \in \mathbb{N}^{<\infty}$ and $n_{0} \in \mathbb{N}$ with $\min E>\max (A \cup B)$ and $n_{0}>\max E$ such that

$$
w(E) \leqslant w(B)<w(E)+w_{n_{0}}<2 w(B) .
$$

Set $F:=E \cup\left\{n_{0}\right\}$. Then $w(E) \leqslant w(B)<w(F)<2 w(B)$. Let $\varepsilon>0$. Applying the $w$-semi-greedy condition to

$$
x=(1+\varepsilon) \sum_{n \in B} \pm e_{n}+\sum_{n \in E} e_{n}
$$

yields scalars $\left(a_{n}\right)_{n \in B}$ such that

$$
\begin{aligned}
\left\|\sum_{n \in B} a_{n} e_{n}+\sum_{n \in E} e_{n}\right\| & \leqslant \bar{K} \sigma_{w(B)}^{w}(x) \\
& \leqslant \bar{K}(1+\varepsilon)\left\|\sum_{n \in B} \pm e_{n}\right\|
\end{aligned}
$$

since $w(E) \leqslant w(B)$. Hence

$$
\left\|\sum_{n \in E} e_{n}\right\| \leqslant 2 \beta\left\|\sum_{n \in B} a_{n} e_{n}+\sum_{n \in E} e_{n}\right\| \leqslant 2 \beta \bar{K}(1+\varepsilon)\left\|\sum_{n \in B} \pm e_{n}\right\| .
$$

Since $\varepsilon>0$ is arbitrary,

$$
\left\|\sum_{n \in E} e_{n}\right\| \leqslant 2 \beta \bar{K}\left\|\sum_{n \in B} \pm e_{n}\right\| .
$$

Similarly, using the fact that $w(A) \leqslant w(B)<w(F)$, the $w$-semi-greedy condition applied to $y=\sum_{n \in A} \pm e_{n}+(1+\varepsilon) \sum_{n \in F} e_{n}$ yields

$$
\left\|\sum_{n \in A} \pm e_{n}\right\| \leqslant \beta \bar{K}\left\|\sum_{n \in F} e_{n}\right\| \text {. }
$$

Finally,

$$
\begin{aligned}
\left\|\sum_{n \in A} \pm e_{n}\right\| & \leqslant \beta \bar{K}\left\|\sum_{n \in F} e_{n}\right\| \\
& \leqslant \beta \bar{K}\left(\left\|\sum_{n \in E} e_{n}\right\|+1\right) \\
& \leqslant \beta \bar{K}\left(2 \beta \bar{K}\left\|\sum_{n \in B} \pm e_{n}\right\|+1\right) \\
& \leqslant\left(2 \beta^{2} \bar{K}^{2}+\beta \bar{K}\right)\left\|\sum_{n \in B} \pm e_{n}\right\| .
\end{aligned}
$$


Proposition 4.9. Suppose $0<\inf w_{n} \leqslant \sup w_{n}<\infty$. Then $\left(e_{n}\right)$ is $w$-superdemocratic $\Leftrightarrow\left(e_{n}\right)$ is superdemocratic for the constant weight sequence.

Proof. $\Rightarrow$ : Let $\bar{D}$ be the $w$-superdemocracy constant of $\left(e_{n}\right)$. We may assume that $0<\alpha:=\inf w_{n} \leqslant 1=\sup w_{n}$. Suppose $|A|=|B|$ and, without loss of generality, $w(A) \leqslant w(B)$. Then

$$
\max _{ \pm}\left\|\sum_{n \in A} \pm e_{n}\right\| \leqslant \bar{D} \min _{ \pm}\left\|\sum_{n \in B} \pm e_{n}\right\|
$$

So to prove superdemocracy it suffices to show that

$$
\max _{ \pm}\left\|\sum_{n \in B} \pm e_{n}\right\| \leqslant L \min _{ \pm}\left\|\sum_{n \in A} \pm e_{n}\right\|
$$

for some constant $L$. If $w(B) \leqslant 2 / \alpha$ then $|B| \leqslant 2 / \alpha^{2}$ and so we can take $L=2 / \alpha^{2}$. Suppose $w(B)>2 / \alpha$. Note that $w(A) \geqslant \alpha w(B) \geqslant 2$. Hence we may partition $B$ into $N$ sets $B_{1}, \ldots, B_{N}$ satisfying $w\left(B_{j}\right) \leqslant$ $w(A) \leqslant w\left(B_{j}\right)+1$, and hence $w\left(B_{j}\right) \geqslant w(A) / 2$, with

$$
N \leqslant \frac{w(B)}{w(A) / 2} \leqslant \frac{2}{\alpha} .
$$

Since $\left(e_{n}\right)$ is $w$-superdemocratic and $w\left(B_{j}\right) \leqslant w(A)(1 \leqslant j \leqslant N)$, we have

$$
\begin{aligned}
\max _{ \pm}\left\|\sum_{n \in B} \pm e_{n}\right\| & \leqslant \sum_{j=1}^{N} \max _{ \pm}\left\|\sum_{n \in B_{j}} \pm e_{n}\right\| \\
& \leqslant N \bar{D} \min _{ \pm}\left\|\sum_{n \in A} \pm e_{n}\right\| \\
& \leqslant \frac{2 \bar{D}}{\alpha} \min _{ \pm}\left\|\sum_{n \in A} \pm e_{n}\right\|
\end{aligned}
$$

Hence we can take $L=2 \bar{D} / \alpha$.

$\Leftarrow$ : Let $C$ be the superdemocracy constant (for the constant weight). Suppose $w(A) \leqslant w(B)$. Then $|A| \leqslant|B| / \alpha$. We can partition $A$ into fewer than $1+1 / \alpha$ sets of size at most $|B|$. Hence by the triangle inequality

$$
\max _{ \pm}\left\|\sum_{n \in A} \pm e_{n}\right\| \leqslant \frac{C(1+\alpha)}{\alpha} \min _{ \pm}\left\|\sum_{n \in B} \pm e_{n}\right\| .
$$

So $\left(e_{n}\right)$ is $w$-superdemocratic. 
Remark 4.10. The previous result is sharp in the following sense. Suppose $w$ is a weight sequence satisfying inf $w_{n}=0, \sup w_{n}=1$, and $\sum w_{n}=\infty$. Consider the following norm:

$$
\left\|\sum_{n=1}^{\infty} a_{n} e_{n}\right\|=\sup \left|a_{n}\right| \vee\left(\sum_{n=1}^{\infty} a_{n}^{2} w_{n}\right)^{1 / 2}
$$

Then $\left(e_{n}\right)$ is a normalized basis which is $w$-superdemocratic but not superdemocratic (and hence $\left(e_{n}\right)$ is $w$-greedy but not greedy).

Corollary 4.11. Suppose that $\left(e_{n}\right)$ has no subsequence equivalent to the unit vector basis of $c_{0}$. Then $\left(e_{n}\right)$ is w-almost greedy for some weight $w \Leftrightarrow\left(e_{n}\right)$ is almost greedy.

Proof. By Theorem 2.6 and Proposition 4.7, $\left(e_{n}\right)$ is $w$-almost greedy $\Leftrightarrow$ $\left(e_{n}\right)$ is quasi-greedy and $w$-superdemocratic. Since $\left(e_{n}\right)$ has no subsequence equivalent to the unit vector basis of $c_{0}$, by Proposition 4.5 (parts (iii) and $(i v)), 0<\inf w_{n} \leqslant \limsup w_{n}<\infty$. Hence, by Proposition 4.9, $\left(e_{n}\right)$ is $w$-almost greedy $\Leftrightarrow\left(e_{n}\right)$ is quasi-greedy and superdemocratic $\Leftrightarrow\left(e_{n}\right)$ is almost greedy.

Question 4.12. Does the analogous result holds for semi-greediness? A simple characterization of w-semi-greediness analogous to Theorem 2.6. which could be useful for answering this question, seems to be lacking. A partial answer is given in Corollary 4.16 below.

We turn now to a converse to Theorem 4.3 for spaces of finite cotype. The following lemma is needed.

Lemma 4.13. Suppose $\left(e_{n}\right)$ is $w$-semi-greedy and $0<\inf w_{n} \leqslant \sup w_{n}$. There exists $M<\infty$ such that for all $A \in \mathbb{N}<\infty$ and for all scalars $\left(a_{n}\right)_{n \in A}$, we have

$$
\min _{n \in A}\left|a_{n}\right|\left\|\sum_{n \in A} e_{n}\right\| \leqslant M\left\|\sum_{n \in A} a_{n} e_{n}\right\| .
$$

Proof. We may assume $\sup w_{n}=1$ and $\inf w_{n}=\alpha>0$. By Proposition 4.9, $\left(e_{n}\right)$ is superdemocratic. We may assume $w(A)>2$, for otherwise $|A| \leqslant 2 / \alpha$ and the result is clear. Choose $F$ with $\min F>\max A$ such that $w(F) \leqslant w(A) \leqslant w(F)+1$. Note that

$$
|F| \geqslant w(A)-1 \geqslant \frac{w(A)}{2} \geqslant \frac{\alpha|A|}{2} \text {. }
$$

Hence

$$
\left\|\sum_{n \in F} e_{n}\right\| \geqslant \frac{\alpha}{(2+\alpha) D}\left\|\sum_{n \in A} e_{n}\right\|
$$


where $D$ is the democracy constant. Applying the semi-greedy condition to $x=\sum_{n \in A} a_{n} e_{n}+\left(\min \left|a_{n}\right|\right) \sum_{n \in F} e_{n}$, there exist scalars $\left(c_{n}\right)_{n \in A}$ such that

$$
\begin{aligned}
\left\|\sum_{n \in A} c_{n} e_{n}+\left(\min \left|a_{n}\right|\right) \sum_{n \in F} e_{n}\right\| & \leqslant \bar{K} \sigma_{w(A)}^{w} \\
& \leqslant \bar{K}\left\|\sum_{n \in A} a_{n} e_{n}\right\| .
\end{aligned}
$$

since $w(F) \leqslant w(A)$. Let $\beta$ be the basis constant of $\left(e_{n}\right)$. Hence

$$
\begin{aligned}
\left(\min \left|a_{n}\right|\right)\left\|\sum_{n \in A} e_{n}\right\| & \leqslant\left(\frac{2}{\alpha}+1\right) D\left(\min \left|a_{n}\right|\right)\left\|\sum_{n \in F} e_{n}\right\| \\
& \leqslant 2 \beta\left(\frac{2}{\alpha}+1\right) D\left\|\sum_{n \in A} c_{n} e_{n}+\left(\min \left|a_{n}\right|\right) \sum_{n \in F} e_{n}\right\| \\
& \leqslant 2 \beta\left(\frac{2}{\alpha}+1\right) D \bar{K}\left\|\sum_{n \in A} a_{n} e_{n}\right\| .
\end{aligned}
$$

Let us recall that a Banach space $X$ has cotype $q$, where $2 \leqslant q<\infty$, if there exists a constant $C$ such that

$$
\left(\sum_{j=1}^{n}\left\|x_{j}\right\|^{q}\right)^{\frac{1}{q}} \leqslant C\left(\underset{\varepsilon_{j}= \pm 1}{\mathrm{Ave}_{j=1}}\left\|\sum_{j=1}^{n} \varepsilon_{j} x_{j}\right\|^{q}\right)^{\frac{1}{q}}
$$

for all $x_{1}, \ldots, x_{n} \in X$ and $n \in \mathbb{N}$. The least such constant $C$ is called the cotype $q$-constant $C_{q}(X)$. We say that $X$ has finite cotype if $X$ has cotype $q$ for some $q<\infty$.

The following result and its proof can be extracted from [DKK, p. 76]. It is used below in the proof of Theorem 4.15.

Proposition 4.14. Suppose that $X$ has finite cotype and that $\left(e_{n}\right)$ is superdemocratic. Then, for all $0<\theta<1$, there exists $L(\theta)<\infty$ such that for all $m \geqslant 1$ and for all $x=\sum_{n \in F} a_{n} e_{n}$, where $|F|=m$, and for all $n \geqslant \theta m$, we have $\left\|G_{n}(x)\right\| \leqslant L(\theta)\|x\|$. $(L(\theta)$ also depends on $X$ and on $\left(e_{n}\right)$ but we suppress this dependence.)

Now we can prove the weighted version of [DKK, Theorem 3.6], which provides a partial converse to Theorem 4.3.

Theorem 4.15. Suppose that $X$ has finite cotype and that $\left(e_{n}\right)$ is $w$ semi-greedy. Then $\left(e_{n}\right)$ is w-almost greedy.

Proof. Since $X$ has finite cotype, no subsequence of $\left(e_{n}\right)$ is equivalent to the unit vector basis of $c_{0}$. Hence we may assume that $\sup w_{n}=1$ 
and inf $w_{n}=\alpha>0$. By Proposition 4.9, $\left(e_{n}\right)$ is both $w$-superdemocatic and superdemocratic. Hence, by Theorem 2.6, it suffices to show that $\left(e_{n}\right)$ is quasi-greedy, i.e., that there exists $K<\infty$ such that for all $k \geqslant 1$ and $x \in X,\left\|G_{k}(x)\right\| \leqslant K\|x\|$.

Fix $m \geqslant 1$. Suppose that $x=\sum_{n \in F} a_{n} e_{n}$ with $\|x\|=1$, where $|F|=m$ and $a_{n} \neq 0$ for $n \in F$. Fix $k \geqslant 1$ and let $A:=\{\rho(1), \ldots, \rho(k)\}$. Choose $E \in \mathbb{N}^{<\infty}$ such that $\min E>\max F$ and $w(A) \leqslant w(E) \leqslant$ $w(A)+1$. This implies that

$$
\alpha k=\alpha|A| \leqslant|E| \leqslant \frac{|A|+1}{\alpha}=\frac{k+1}{\alpha} \leqslant \frac{2 k}{\alpha} .
$$

Now let $B:=\{\rho(k+1), \ldots, \rho(k+l))\}$, where $w(A) \leqslant w(B) \leqslant$ $w(A)+1$, so that

$$
\alpha k=\alpha|A| \leqslant|B|=l \leqslant \frac{|A|+1}{\alpha}=\frac{k+1}{\alpha} \leqslant \frac{2 k}{\alpha} .
$$

If $k+l>m$, then $k>\alpha m /(2+\alpha)$, and hence by Proposition 4.14

$$
\left\|G_{k}(x)\right\| \leqslant L(\alpha /(2+\alpha))\|x\| .
$$

So we may assume $k+l \leqslant m$.

Fix $\varepsilon>0$ and consider

$$
y:=\sum_{n \in F \backslash A} a_{n} e_{n}+\left(\left|a_{\rho(k)}\right|+\varepsilon\right)\left(\sum_{n \in E} e_{n}\right) .
$$

Since $w(A) \leqslant w(E)$, we have

$$
\sigma_{w(E)}^{w}(y) \leqslant\left\|x+\left(\left|a_{\rho(k)}\right|+\varepsilon\right)\left(\sum_{n \in E} e_{n}\right)\right\| \leqslant 1+\left(\left|a_{\rho(k)}\right|+\varepsilon\right)\left\|\sum_{n \in E} e_{n}\right\| .
$$

Since $\left(e_{i}\right)$ is semi-greedy there exist scalars $\left(c_{n}\right)(n \in E)$ such that

$$
\left\|\sum_{n \in F \backslash A} a_{n} e_{n}+\sum_{n \in E} c_{n} e_{n}\right\| \leqslant \bar{K} \sigma_{w(E)}^{w}(y) .
$$

Since $\max F<\min E$ and $\varepsilon>0$ is arbitrary, (4.4) and (4.5) yield

$$
\left\|\sum_{n \in F \backslash A} a_{n} e_{n}\right\| \leqslant \beta \bar{K}\left(1+\left|a_{\rho(k)}\right|\left\|\sum_{n \in E} e_{n}\right\|\right)
$$

where $\beta$ is the basis constant. Hence

$$
\left\|G_{k}(x)\right\|=\left\|x-\sum_{n \in F \backslash A} a_{n} e_{n}\right\| \leqslant 1+\beta \bar{K}\left(1+\left|a_{\rho(k)}\right|\left\|\sum_{n \in E} e_{n}\right\|\right) .
$$

Now consider

$$
z:=x-\sum_{n \in A} a_{n} e_{n} .
$$


Then $\left\|\sigma_{w(B)}^{w}(z)\right\| \leqslant\|x\|=1$, since $w(A) \leqslant w(B)$. Since $\left(e_{n}\right)$ is semigreedy there exist scalars $\left(\bar{c}_{n}\right)(n \in B)$ such that

$$
\left\|z-\sum_{n \in B} \bar{c}_{n} e_{n}\right\| \leqslant \bar{K}\left\|\sigma_{w(B)}^{w}(z)\right\| \leqslant \bar{K} .
$$

Hence

$$
\left\|\sum_{n \in A} a_{n} e_{n}+\sum_{n \in B} \bar{c}_{n} e_{n}\right\|=\left\|x-\left(z-\sum_{n \in B} \bar{c}_{n} e_{n}\right)\right\| \leqslant\|x\|+\bar{K}=1+\bar{K} .
$$

Let $\bar{B}:=\left\{n \in B:\left|\bar{c}_{n}\right| \geqslant\left|a_{\rho(k)}\right|\right\}$. Then

$$
\sum_{n \in A} a_{n} e_{n}+\sum_{n \in \bar{B}} \bar{c}_{n} e_{n}=G_{s}\left(\sum_{n \in A} a_{n} e_{n}+\sum_{n \in B} \bar{c}_{n} e_{n}\right)
$$

for some $k \leqslant s \leqslant k+l \leqslant(1+2 / \alpha) k$. Hence Proposition 4.14 and (4.7) yield

$$
\left\|\sum_{n \in A} a_{n} e_{n}+\sum_{n \in \bar{B}} \bar{c}_{n} e_{n}\right\| \leqslant L(\alpha /(\alpha+2))(1+\bar{K})
$$

On the other hand, by Lemma 4.13 there exists $M<\infty$, depending only on $\left(e_{n}\right)$, such that

$$
\left|a_{\rho(k)}\right|\left\|\sum_{n \in A \cup \bar{B}} e_{n}\right\| \leqslant M\left\|\sum_{n \in A} a_{n} e_{n}+\sum_{n \in \bar{B}} c_{n} e_{n}\right\| .
$$

From (4.3), we obtain

$$
|E| \leqslant \frac{2}{\alpha} k \leqslant \frac{2}{\alpha}|A \cup \bar{B}|,
$$

and hence, since $\left(e_{n}\right)$ is superdemocratic (with constant $D$, say),

$$
\left\|\sum_{n \in E} e_{n}\right\| \leqslant D\left(\frac{2}{\alpha}+1\right)\left\|\sum_{n \in A \cup \bar{B}} e_{n}\right\| .
$$

Finally, combining (4.6), (4.8), (4.9), and (4.10) we get

$$
\left\|G_{k}(x)\right\| \leqslant 1+\beta \bar{K}+\beta \bar{K} D\left(\frac{2}{\alpha}+1\right) M L\left(\frac{\alpha}{\alpha+2}\right)(1+\bar{K}) .
$$

Combining Corollary 4.11 and Theorem 4.15 we get a partial answer to Question 4.12.

Corollary 4.16. Suppose that $X$ has finite cotype. Then $\left(e_{n}\right)$ is wsemi-greedy for some weight $w \Leftrightarrow\left(e_{n}\right)$ is semi-greedy. 


\section{5. $w$-ALMOST GREEDY BASES WHEN $w \in c_{0}$}

Proposition 5.1. Assume $w \in c_{0}$ and is nonincreasing. Let $\left(e_{n}\right)_{n=1}^{\infty}$ be a normalized D-w-democratic basis for a (real or complex) Banach space $X$. Then for every $m \in \mathbb{N}$ there exists $N \in \mathbb{N}$ so that if $A \in \mathbb{N}^{m}$ with $N \leqslant \min A$ then

$$
\left\|\sum_{n \in A} e_{n}\right\| \leqslant D
$$

If furthermore $w \in \ell_{1}$, we can choose $M \in \mathbb{N}$ such that (5.1) holds for all $A \in \mathbb{N}<\infty$ with $M \leqslant \min A$.

Proof. Observe that

$$
\lim _{k \rightarrow \infty} \sum_{i=k}^{k+m-1} w_{i}=0,
$$

so that we can find $N \in \mathbb{N}$ satisfying

$$
w(A)=\sum_{i \in A} w_{i} \leqslant \sum_{i=N}^{N+m-1} w_{i}<w_{1}=w(\{1\})
$$

for all $A \in \mathbb{N}^{m}$ satisfying $N \leqslant \min A$. By $D$-w-democracy this means

$$
\left\|\sum_{n \in A} e_{n}\right\| \leqslant D\left\|x_{1}\right\|=D
$$

This proves the first part of the proposition.

Next, assume $w \in \ell_{1}$, and let $M \in \mathbb{N}$ be such that

$$
\sum_{i=M}^{\infty} w_{i}<w_{1}
$$

and hence $w(A)<w(\{1\})$ for all $A \in \mathbb{N}<\infty$ with $M \leqslant \min A$. Then we have (5.1) as before.

Remark 5.2. It is easy to see from the proof of Proposition 5.1 that if $w \in c_{0}$ is not assumed to be nonincreasing, we can still find a constant $D$ such that for any $m \in \mathbb{N}$ there is a positive integer $N \in \mathbb{N}$ satisfying the property that if $k \geqslant N$ then there exists $A \in \mathbb{N}^{m}$ with $k \leqslant A$ and $\left\|\sum_{n \in A} e_{n}\right\| \leqslant D$.

Corollary 5.3. Let $w \in c_{0}$. If $\left(e_{n}\right)_{n=1}^{\infty}$ is a basis for a (real or complex) Banach space which is both $w$-democratic and spreading then it is equivalent to the canonical basis for $c_{0}$.

Proof. Assume $\left(e_{n}\right)_{n=1}^{\infty}$ is both $w$-democratic and spreading. By Proposition 5.1 and Remark 5.2 there is a constant $C$ such that

$$
\left\|\sum_{n \in A} e_{n}\right\| \leqslant C
$$


for arbitrarily large $A \in \mathbb{N}<\infty$.

We claim that $\left(e_{n}\right)_{n=1}^{\infty}$ is unconditional. Indeed, every conditional spreading basis dominates the summing basis for $c_{0}$ by [FOSZ, p4]. Let $C^{\prime}$ be the domination constant. Then we would have

$$
|A| \leqslant C^{\prime}\left\|\sum_{n \in A} e_{n}\right\| \leqslant C C^{\prime}
$$

which is impossible for sufficiently large $A$. This proves the claim.

Let $U$ be the unconditional constant for $\left(e_{n}\right)_{n=1}^{\infty}$, and select any $\left(a_{n}\right)_{n=1}^{\infty}$ with support in $A$. Then

$$
\frac{1}{2 \beta M} \sup _{n \in A}\left|a_{n}\right| \leqslant\left\|\sum_{n \in A} a_{n} e_{n}\right\| \leqslant U C \sup _{n \in A}\left|a_{n}\right|,
$$

where $M=\sup _{n \in \mathbb{N}}\left\|e_{n}\right\|$. By the spreading property, this means $\left(e_{n}\right)_{n=1}^{\infty}$ is equivalent to the canonical basis for $c_{0}$.

Definition 5.4. A basis $\left(e_{n}\right)_{n=1}^{\infty}$ is conservative with constant $\boldsymbol{C}$ whenever

$$
\left\|\sum_{n \in A} e_{n}\right\| \leqslant C\left\|\sum_{n \in B} e_{n}\right\|
$$

for all $A, B \in \mathbb{N}^{<\infty}$ satisfying $|A| \leqslant|B|$ and $A<B$.

It is known (see [DKKT, Theorem 3.4]) that a basis is partiallygreedy if and only if it is both quasi-greedy and conservative.

Proposition 5.5. Assume $w \in c_{0}$. If a normalized basis $\left(e_{n}\right)_{n=1}^{\infty}$ is both $C$-conservative and $D$-w-democratic then it is $2 C D \beta$-democratic, where $\beta$ is the basis constant for $\left(e_{n}\right)_{n=1}^{\infty}$. Furthermore,

$$
\left\|\sum_{n \in A} e_{n}\right\| \leqslant C D \quad \text { for all } A \in \mathbb{N}^{<\infty}
$$

Proof. Let $A, B \in \mathbb{N}<\infty$ with $|A| \leqslant|B|$. By Proposition 5.1 and Remark 5.2 we can find $\Gamma \in \mathbb{N}<\infty$ with $|B| \leqslant|\Gamma|$ and $A \cup B<\Gamma$, and satisfying

$$
\left\|\sum_{n \in \Gamma} e_{n}\right\| \leqslant D
$$

Note that $|A| \leqslant|\Gamma|$ and $A<\Gamma$ so that by $C$-conservativeness we now have

$$
\left\|\sum_{n \in A} e_{n}\right\| \leqslant C\left\|\sum_{n \in \Gamma} e_{n}\right\| \leqslant C D .
$$

By taking the difference of partial sum projections we also have

$$
C D \leqslant 2 C D \beta\left\|\sum_{n \in B} e_{n}\right\| .
$$


Theorem 5.6. Assume $w \in c_{0}$. A quasi-greedy basis $\left(e_{n}\right)_{n=1}^{\infty}$ for a real Banach space is both conservative and $w$-democratic if and only if it is equivalent to the canonical basis for $c_{0}$.

Remark 5.7. It is clear that greediness implies neither $w$-almostgreediness nor $w$-democracy when $w \in c_{0}$, as for instance $\ell_{p}$ is greedy but not $w$-democratic in this case. Theorem 5.6 shows that $w$-almostgreediness implies partially-greediness (or almost-greediness, or greediness) if and only if we have

$$
\left(e_{n}\right)_{n=1}^{\infty} \text { is } w \text {-almost greedy } \Leftrightarrow\left(e_{n}\right)_{n=1}^{\infty} \approx\left(f_{n}\right)_{n=1}^{\infty},
$$

where $\left(f_{n}\right)_{n=1}^{\infty}$ denotes the canonical basis of $c_{0}$.

Proof of Theorem 5.6. The "if" part is trivial, so we need only prove the "only if" part. We now assume $\left(e_{n}\right)_{n=1}^{\infty}$ is quasi-greedy, conservative, and $w$-democratic. Then by Proposition 5.5, we can find a constant $C$ with

$$
\left\|\sum_{n \in A} e_{n}\right\| \leqslant C \quad \text { for all } A \in \mathbb{N}^{<\infty}
$$

Meanwhile, by quasi-greediness together with Proposition [2.2, and making $C$ larger if necessary, we have

$$
\left\|\sum_{n \in A} a_{n} e_{n}\right\| \leqslant 2 C\left\|\sum_{n \in A} e_{n}\right\| \cdot\left\|\left(a_{n}\right)_{n \in A}\right\|_{\infty}
$$

for any $A \in \mathbb{N}^{<\infty}$. Also, if $\beta$ is the basis constant for $\left(e_{n}\right)_{n=1}^{\infty}$, then by looking at the difference of partial sum projections we obtain

$$
\left\|\left(a_{n}\right)_{n \in A}\right\|_{\infty} \leqslant 2 \beta\left\|\sum_{n \in A} a_{n} e_{n}\right\| .
$$

Putting all these inequalities together, it follows that $\left(e_{n}\right)_{n=1}^{\infty}$ is equivalent to $c_{0}$.

Theorem 5.8. Assume $w \in c_{0}$. If $\left(e_{n}\right)_{n=1}^{\infty}$ is a normalized w-almost greedy basis for a real Banach space $X$, then for some $1 \leqslant C<\infty$ and every $m \in \mathbb{N}$ there exists $N \in \mathbb{N}$ so that $\left(e_{n}\right)_{n=N+1}^{N+m} \approx_{C}\left(f_{n}\right)_{n=1}^{m}$, where $\left(f_{n}\right)_{n=1}^{\infty}$ is the canonical basis for $c_{0}$. Furthermore, we can choose $C$ such that every subsequence of $\left(e_{n}\right)_{n=1}^{\infty}$ admits a further subsequence which is $C$-equivalent to the canonical basis for $c_{0}$.

Proof. Let $\beta$ be the basis constant for $\left(e_{n}\right)_{n=1}^{\infty}$. We showed in the previous section that every $w$-almost greedy basis is both quasi-greedy and $w$-democratic. Fix $m \in \mathbb{N}$, and let $N \in \mathbb{N}$ be as in Proposition 
5.1, which we can do by $w$-democracy. We claim that there exists a constant $C$ such that

$$
\frac{1}{2 \beta} \sup _{n=N+1, \cdots, N+m}\left|a_{n}\right| \leqslant\left\|\sum_{n=N+1}^{N+m} a_{n} e_{n}\right\| \leqslant C \sup _{n=N+1, \cdots, m+N}\left|a_{n}\right|
$$

for all $\left(a_{n}\right)_{n=1}^{\infty} \in c_{0}$. In the above, the first inequality follows from taking the difference of basis projections, and the second inequality for a constant $C$ follows from combining Propositions 2.2 and 5.1. Without loss of generality we may assume $C \geqslant 2 \beta$, completing the proof of the first part of the theorem.

Now let us prove the "furthermore" part. Note that by Remark 1.4. every subsequence of $\left(e_{n}\right)_{n=1}^{\infty}$ admits a further subsequence which is $C$ - $w^{\prime}$-democratic with $w^{\prime} \in \ell_{1}$. Thus, it is enough to show that $\left(e_{n}\right)_{n=M}^{\infty} \approx_{C} c_{0}$ for some $M \in \mathbb{N}$ whenever $w \in \ell_{1}$.

By Proposition 5.1 we can find $M \in \mathbb{N}$ with

$$
\left\|\sum_{n \in A} e_{n}\right\| \leqslant C
$$

for all $A \in \mathbb{N}^{<\infty}$ with $M \leqslant \min A$. Thus, as before we can find $C \geqslant 2 \beta$ with

$$
\frac{1}{2 \beta}\left\|\left(a_{n}\right)_{n \in A}\right\|_{\infty} \leqslant\left\|\sum_{n \in A} a_{n} e_{n}\right\| \leqslant C\left\|\left(a_{n}\right)_{n \in A}\right\|_{\infty} .
$$

Corollary 5.9. If $w \in c_{0}$ then every $w$-almost greedy basis of a real Banach space is weakly null.

Proof. Suppose $\left(e_{n}\right)_{n=1}^{\infty}$ is not weakly null. Then there exists $f \in X^{*}$ such that $f\left(e_{n}\right) \not \rightarrow 0$. Now find a subsequence such that $\left|f\left(x_{n_{k}}\right)\right| \rightarrow \delta>$ 0 . Then $\left(x_{n_{k}}\right)_{k=1}^{\infty}$ contains no weakly null subsequence. In particular, it contains no subsequence equivalent to the $c_{0}$ basis. By Theorem 5.8 it is not $w$-almost greedy.

\section{REFERENCES}

[BB] P. Berna and O. Blasco, The best $m$-term approximation with respect to polynomials with constant coefficients, preprint.

[CDH] A. Cohen, R. Devore, R. Hochmuth, Restricted nonlinear approximation, Constr. Approx., 16 (2000), no. 1, 85-113.

[DKK] S.J. Dilworth, N.J. Kalton, Denka Kutzarova, On the existence of almost greedy bases in Banach spaces, Studia Math. 159 (2003), 67-101.

[DKKT] S.J. Dilworth, N.J. Kalton, Denka Kutzarova, V.N. Temlyakov, The Thresholding Greedy Algorithm, Greedy Bases, and Duality, Constructive Approximation 19 (2003), no. 4, 575-597. 
[DS-BT] S.J. Dilworth, M. Soto-Bajo, and V.N. Temlyakov, Quasi-greedy bases and Lebesgue-type inequalities, IMI Preprints, 02 (2012), 1-44.

[FOSZ] D. Freeman, E. Odell, B. Sari, B. Zheng. On spreading sequences and asymptotic structures, (preprint, 2016). arXiv:1607.03587v1 [math.FA]

[GHO] G. Garrigos, E. Hernandes, and T. Oikhberg, Lebesgue-type inequalities for quasi-greedy bases, Constr. Approx. 38 (2013), 447-470.

[KPT] G. Kerkyacharian, D. Picard and V.N. Temlyakov, Some inequalities for the tensor product of greedy bases and weight-greedy bases, East J. Approx. 12 (2006), 103-118.

[KT] S. V. Konyagin and V. N. Temlyakov, A remark on greedy approximation in Banach spaces, East J. Approx. 5 (1999), no. 3, 365-379.

[T1] V. N. Temlyakov, Greedy Approximation, Cambridge University Press, 2011.

[T2] V. N. Temlyakov, Sparse Approximation with Bases, Advanced Courses in Mathematics CRM Barcelona, Birkhäuser, 2015.

[W] P. Wojtaszczyk, Greedy algorithm for general biorthogonal systems, J. Approx. Theory 107 (2000), 293-314.

Department of Mathematics, University of South Carolina, Columbia, SC, USA

E-mail address: dilworth@math.sc.edu

Department of Mathematics, University of Illinois at Urbana-Champaign, Urbana, IL, USA; Institute of Mathematics and Informatics, BulgarIAN ACADEMY OF SCIENCES

E-mail address: denka@math.uiuc.edu

Department of Mathematics, University of South Carolina, Columbia, SC, USA; Steklov Institute of Mathematics, Moscow, Russia; Lomonosov Moscow State University, Moscow, Russia

E-mail address: temlyak@math.sc.edu

Department of Mathematical Sciences, Northern Illinois UniverSity, Dekalb, IL, USA

E-mail address: benwallis@live.com 